\title{
Thermal and Dynamic Effects in Langevin Simulation of Hysteresis in Nanoscale Pillars
}

\author{
Gregory Brown ${ }^{a}$, M.A. Novotny ${ }^{a}$, and P.A. Rikvold ${ }^{a, b}$ \\ a School of Computational Science and Information Technology, Florida State University, \\ Tallahassee, Florida 32306-4120 \\ ${ }^{b}$ Center for Materials Research and Technology, and Department of Physics, Florida State \\ University, Tallahassee, Florida 32306-4350
}

(November 5, 2018)

\begin{abstract}
Dynamic quantities related to hysteresis have been measured in micromagnetic simulations of single-domain nanoscale magnets at nonzero temperature. The hysteresis-loop area and magnetization-field correlation display the characteristics of resonance, and the resonance frequency is found to be temperature dependent. The period-averaged magnetization displays symmetry breaking at high frequencies.
\end{abstract}

Keywords: thermal, hysteresis, resonance, micromagnetic

The common theme in hysteretic systems is a nonlinear, irreversible response that lags behind an applied force. While different physical mechanisms may cause this behavior, in many interesting situations it results from a system with two local free-energy minima separated by a free-energy maximum. For instance, the crystalline or shape anisotropy of a magnetic particle may create a barrier that significantly interferes with the reversal mechanism whereby the magnetization changes from the unfavorable alignment antiparallel to the applied field to the equilibrium alignment parallel to the field. Historically, hysteresis experiments have been conducted under quasistatic conditions that minimize thermal and dynamic effects. With new technology making applications of magnetization reversal of nanoscale magnets on the nanosecond time scale important, a thorough understanding of these effects is needed. Here we present results from dynamic micromagnetic modeling of hysteresis in nanoscale pillars at nonzero temperatures. 
Our numerical model is based on iron nanopillars fabricated using STM-assisted chemical vapor deposition [1,2]. Following the one-dimensional model for magnetic pillars discussed by Boerner and Bertram [3], the nanoscale magnets are decomposed into a one-dimensional stack of 17 cubes with side $7.2 \mathrm{~nm}$. Each cube has a magnetization density $\mathbf{M}\left(\mathbf{r}_{i}, t\right)$ and a local magnetic field $\mathbf{H}\left(\mathbf{r}_{i}, t\right)$, with $\mathbf{r}_{i}$ the center of the $i$-th cube. The damped precessional motion of each magnetization vector is given by the Landau-Lifshitz-Gilbert (LLG) equation 4,5

$$
\frac{\mathrm{d} \mathbf{M}\left(\mathbf{r}_{i}\right)}{\mathrm{d} t}=\frac{\gamma_{0}}{1+\alpha^{2}} \mathbf{M}\left(\mathbf{r}_{i}\right) \times\left(\mathbf{H}\left(\mathbf{r}_{i}\right)-\frac{\alpha}{M_{s}} \mathbf{M}\left(\mathbf{r}_{i}\right) \times \mathbf{H}\left(\mathbf{r}_{i}\right)\right)
$$

where the electron gyromagnetic ratio is $\gamma_{0}=1.76 \times 10^{7} \mathrm{~Hz} / \mathrm{Oe}$ [5], and $\alpha=0.1$ is a phenomenological damping parameter. The numerical details have been given elsewhere [6,7],8]. Parameters corresponding to bulk iron were used: saturation magnetization density $M_{s}=$ $1700 \mathrm{emu} / \mathrm{cm}^{3}$ and exchange length $l_{\mathrm{e}}=3.6 \mathrm{~nm}$. The local magnetic field $\mathbf{H}\left(\mathbf{r}_{i}, t\right)$ is a linear superposition of terms corresponding to exchange, magnetostatic interactions, the applied field, and a stochastic field representing thermal fluctuations. The latter term is governed by the fluctuation-dissipation theorem, for which we use the form derived for isolated magnetic particles [4, 8]. The stochastic integration [8] of Eq. (11) was performed using a first-order Euler scheme with $\Delta t=5 \times 10^{-14} \mathrm{~s}$.

Thermal fluctuations are essential in this model because the free-energy barrier can be surmounted using thermal energy momentarily "borrowed" from the surroundings. For the spatially nonuniform reversal mechanisms considered here [3, 6, 8], this barrier crossing occurs at the ends of the pillar. The observed hysteretic behavior depends on the interplay of three time scales: the mean nucleation time, the time required for the subsequent growth of the nucleated volumes to fill the particle, and the period of the applied field.

To simulate hysteresis loops, an external field $\mathbf{H}=\hat{\mathbf{z}} H_{0} \cos (2 \pi \nu t)$ is applied, with $H_{0}=2000$ Oe. The results are presented for the reduced quantities $m(t)$, the $z$-component of the magnetization for the entire magnet, and $h(t)$, the applied magnetic field, both normalized to have maximum values of unity. 
The correlation between $m$ and $h$ is measured by

$$
B=\frac{\pi \nu}{2} \oint m(t) h(t) \mathrm{d} t,
$$

where the integral is taken over one hysteresis loop. The results are presented vs. $\nu$ in Fig. 1 for $T=20 \mathrm{~K}$ and $100 \mathrm{~K}$. The bars shown for $T=100 \mathrm{~K}$ are not error bars, instead they represent the standard deviation of the probability density for $B$. An example of $m(t)$ is shown as the solid curve in the inset of Fig. 1, along with $h(t) / 10$ which appears as the dotted curve. In the high-frequency limit the magnetization usually does not switch, and $B$ is near zero. At low frequencies the magnetization switches nearly every period, and $B$ approaches unity. The quantity $B$ corresponds to the reactive part of the nonlinear response function [9], and the lowest-frequency zero crossing of $B$ in Fig. 1 is associated with resonance.

The dissipative part of the nonlinear response is the hysteresis-loop area,

$$
A=-\frac{1}{4} \oint m(h) \mathrm{d} h .
$$

The measured average values of $A$ are shown vs. $\nu$ in Fig. 2 for both temperatures. At high frequencies the magnetization is unable to respond to the applied field, and the hysteresis loop area is nearly zero as most of the time $m(t)$ oscillates with a small amplitude about one or the other of the degenerate values of the zero-field magnetization. In the low-frequency (or quasi-static) limit the magnetization usually switches at small values of $h(t)$, and as a result $A$ is again near zero. At intermediate frequencies $A$ displays a maximum near the resonance frequency. The maximum in $A$ and the low-frequency zero crossing in $B$ agree to about $15 \%$. The temperature dependence of the results is mostly due to the different average nucleation times associated with the different temperatures. Similar simulations in two-dimensional Ising magnets have shown that the phenomena seen here are associated with stochastic resonance [9].

Another interesting quantity to consider is the period-averaged magnetization,

$$
Q=\nu \oint m(t) \mathrm{d} t
$$


which corresponds to a dynamic order parameter for the system. Histograms are shown for several applied-field frequencies at $T=100 \mathrm{~K}$ in Fig. 3. At high frequencies the histogram has two maxima, corresponding to the magnetization being oriented predominantly in the positive or negative direction during the entire hysteresis loop. At low frequencies the histogram is centered around zero since the magnetization switches during every half-period of the applied field. Previously, a phase transition in this dynamic order parameter has been seen in Ising models of nanoscale magnets [10,11, 12, 13] when many nucleation events contribute to each reversal of the magnetization. In these models, this phase transition occurs near the frequency where $B(\nu)$ has its high-frequency zero crossing. However, the detailed analysis and large statistical sampling required to make such a determination has not been performed for the present simulations.

In summary, micromagnetic simulations of nanoscale magnets at nonzero temperatures and gigahertz frequencies display the characteristics of temperature-dependent resonance. Future work exploring the connections to stochastic resonance and dynamic phase transitions is planned.

This work was partially supported by the Ames Laboratory, which is operated for the U.S. Department of Energy by Iowa State University under Contract No. W-7405-82, by the Laboratory Directed Research and Development Program of Oak Ridge National Laboratory, managed by UT-Battelle, LLC for the U.S. Department of Energy under Contract No. DE-AC05-00OR22725, and by the U.S. National Science Foundation through grant DMR9871455. Computer resources were provided by the Department of Physics at Florida State University.

[1] S. Wirth, M. Field, D.D. Awschalom, and S. von Molnár, Phys. Rev. B 57 (1998) R14028.

[2] S. Wirth, M. Field, D.D. Awschalom, and S. von Molnár, J. Appl. Phys. 85 (1999) 5249. 
[3] E.D. Boerner and H.N. Bertram, IEEE Trans. Magn. 33 (1997) 3052.

[4] W.F. Brown, Phys. Rev. 130 (1963) 1677.

[5] A. Aharoni, Introduction to the Theory of Ferromagnetism (Clarendon, Oxford, 1996).

[6] G. Brown, M.A. Novotny, P.A. Rikvold, J. Appl. Phys. 87 (2000) 4792.

[7] G. Brown, M.A. Novotny, P.A. Rikvold, J. Appl. Phys. (in press) cond-mat/0010184.

[8] G. Brown, M.A. Novotny, P.A. Rikvold, submitted to Phys. Rev. B cond-mat/0101477.

[9] S.W. Sides, P.A. Rikvold, and M.A. Novotny, Phys. Rev. E 57 (1998) 6512.

[10] S.W. Sides, P.A. Rikvold, and M.A. Novotny, Phys. Rev. Lett. 81 (1998) 834.

[11] S.W. Sides, P.A. Rikvold, and M.A. Novotny, Phys. Rev. E 59 (1999) 2710.

[12] G. Korniss, C.J. White, P.A. Rikvold, and M.A. Novotny, Phys. Rev. E 63 (2001) 016120.

[13] H. Fujisaka, H. Tutu, and P.A. Rikvold, Phys. Rev. E 63 (2001) 036109. 


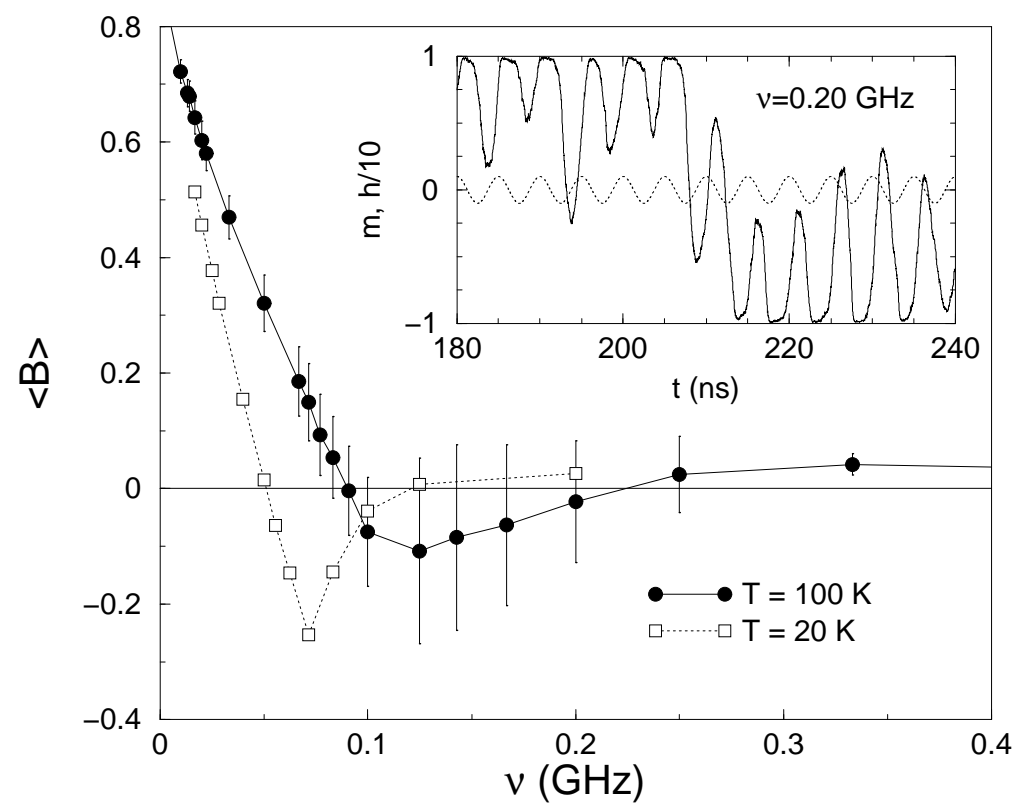

FIG. 1. The correlation $B$, defined in Eq. (2), between the normalized magnetization $m$ and the normalized applied field $h$, shown vs. the applied field frequency $\nu$, for $T=20 \mathrm{~K}$ and $100 \mathrm{~K}$. The bars are not error bars; instead, they represent the standard deviation of $B$ at $T=100 \mathrm{~K}$. The standard error in the measured mean is smaller than the symbol size. As $B$ is the reactive part of the nonlinear system response, the first zero crossing is taken as a sign of resonance. A particular example of $m(t)$ (solid curve) and $h(t) / 10$ (dotted curve) for $T=100 \mathrm{~K}$ and $\nu=0.2 \mathrm{GHz}$ is shown for several hysteresis-loop periods in the inset. 


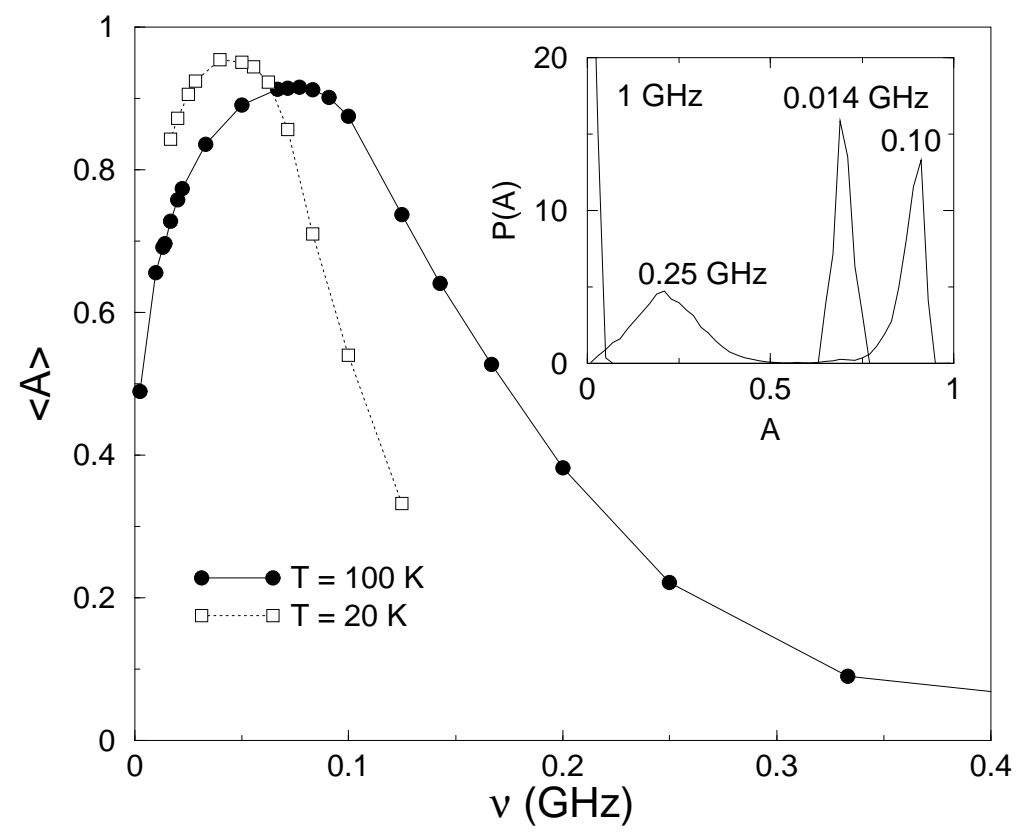

FIG. 2. The mean hysteresis-loop area $A$, defined in Eq. (3), vs. the applied field frequency $\nu$ for $T=20 \mathrm{~K}$ and $100 \mathrm{~K}$. The standard error in the measured mean is smaller than the symbol size. The maximum in $A$, which is the dissipative part of the nonlinear response function, is associated with resonance. The temperature-dependent resonance frequencies agree well with those indicated in Fig. 1. Histograms of $A$ for several different applied field frequencies at $100 \mathrm{~K}$ are shown in the inset. 


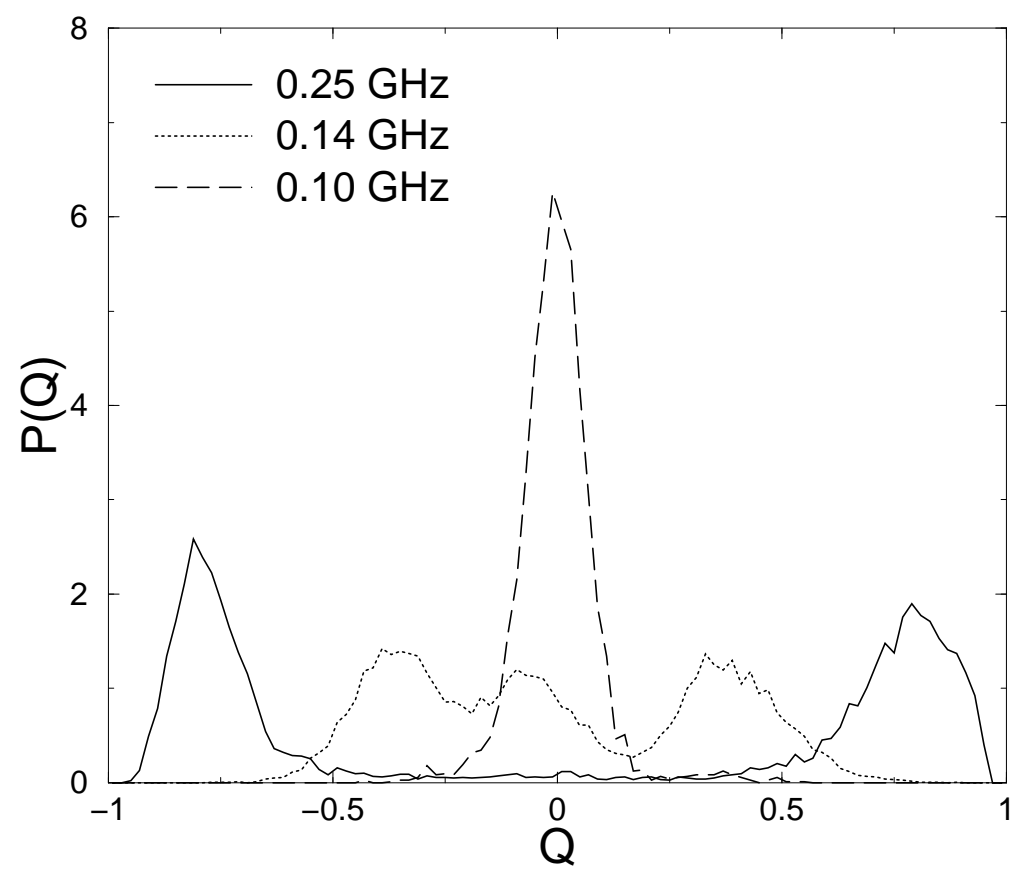

FIG. 3. Histogram of the period-averaged magnetization $Q$, defined in Eq. (四), for applied field frequencies of $0.25,0.14$, and $0.1 \mathrm{GHz} . Q$ is a dynamic order parameter for the system. The two peaks a the highest frequency indicate a broken symmetry state. 